\title{
Propuesta lúdica: un enfoque hacia el fortalecimiento del pensamiento aleatorio
}

Artículo de Investigación

Fecha de Recepción: 8 diciembre 2017.

Fecha de Aprobación: 30 abril 2018. Martha Raquel Martínez Camargo*
Patricia Consuelo Martinez Camargo **

\section{Resumen}

Este documento presenta el desarrollo de una primera fase de investigación de un estudio cualitativo descriptivo realizado con los estudiantes de grado segundo y tercero de básica primaria de una institución educativa de carácter técnico, ubicado en municipio de Duitama. En primer lugar, se destacan los aportes de los trabajos realizados sobre el problema de investigación, seguido de los sustentos teóricos referidos al desarrollo del pensamiento aleatorio y sistemas de datos, un componente esencial en el mundo de las matemáticas, estrategias didácticas y lúdica. Se describe le desarrollo metodológico, mencionando el tipo de estudio, el método, los instrumentos y las técnicas de recolección y análisis de datos. Finalmente se acentúa que el taller diagnóstico aplicado muestra que para los niños es más factible identificar conceptos de cantidad y calidad que los conceptos de variable cuantitativa y cualitativa; asimismo, se les dificulta el paso de la tabla de frecuencias al diagrama de barras, al igual que la relación existente entre la característica del eje horizontal y la magnitud en eje vertical.

Palabras clave: Pensamiento aleatorio, sistemas de datos, magnitudes, lúdica y estrategias didácticas.
* Instituto Técnico Industrial Rafael Reyes Boyacá - Colombia tuchis181@gmail.com ** Instituto Técnico Industrial Rafael Reyes Boyacá - Colombia martinezpatricia32@gmail. com

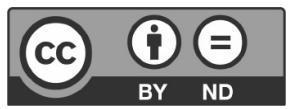




\section{Introducción}

En la actualidad las políticas públicas del ámbito educativo en Colombia, son expedidas por el Ministerio de Educación Nacional (MEN) y buscan el mejoramiento del nivel educativo en todo el país; en tal sentido se han venido implementando los estándares básicos de competencias en las diferentes áreas del conocimiento, así como la evaluación de conocimientos y habilidades fundamentales de los educandos y, de los educadores los saberes disciplinares y pedagógicos, entre otros.

Sumado a lo anterior el programa Todos a Aprender (MEN, 2006) muestra en el documento: "Introducción al desarrollo del pensamiento aleatorio y sistemas de datos", la importancia de promover el pensamiento aleatorio, puesto que proporciona al niño elementos para la toma de decisiones y da la posibilidad de explorar de manera investigativa las relaciones que tiene con otros pensamientos como el numérico y la resolución de problemas.

Con respecto a diversidad de estrategias que favorecen el desarrollo de competencias en el pensamiento aleatorio y con el propósito de interactuar con la didáctica, la metodología y la valoración como principales protagonistas en la educación a partir de las experiencias de aprendizaje, se abordan estudios desde diferentes ámbitos, relacionados con los modos de apropiación del conocimiento por parte de los estudiantes y brindan la oportunidad de mejorar los procesos de predicción e intuición presentes en el pensamiento aleatorio.

Por su parte Camacho y Medina (2015), al igual que Chamarro y Pinzón (2013) presentan diversas herramientas pedagógicas acordes a los procesos de enseñanza los cuales se implementan en la vida cotidiana de forma oportuna con metodologías que tienen como objetivo la ejecución de actividades lúdico-pedagógicas que busquen mejorar las habilidades de pensamiento aleatorio y sistemas de datos en los educandos. Se puede evidenciar entonces que el recurso lúdico y didáctico apoya los procesos de este pensamiento teniendo en cuenta el conocimiento estratégico que fortalece la dinámica del pensamiento matemático.

De igual forma, Rentería (2016) expresa que el aprendizaje de las matemáticas debe iniciar desde los primeros años como un proceso que consiga motivar al estudiante hacia la adquisición de conceptos básicos y hábitos que le permitan generalizar, analizar, solucionar problemas, descubrir relaciones y desarrollar sus habilidades e intereses concretos por el área. Luego es viable implementar una herramienta de apoyo que favorezca el proceso a través del desarrollo de competencias relacionadas con el pensamiento aleatorio, habilidades y estrategias lúdicas pedagógicas que propicien el interés y aprendizaje significativo en los estudiantes.

Ahora, en el interés por mejorar los procesos de enseñanza y aprendizaje 
de las matemáticas en los diferentes niveles académicos, en el ámbito nacional se han encontrado diversas investigaciones como la de Jaramillo y Quintero (2015) quienes dan a conocer como la implementación de la lúdica y la utilización de ambientes virtuales de aprendizaje, contribuyen al desarrollo de competencias matemáticas, específicamente del pensamiento aleatorio. Estas estrategias propician ambientes de aprendizaje fundamentados en la lúdica que estimulan al desarrollo del pensamiento aleatorio en los estudiantes y didácticas acompañadas de recursos, herramientas tecnológicas que generan una excelente base pedagógica. A la par, el trabajo de García Quintero (2012) planteo una estrategia didáctica basada en la lúdica para promover el aprendizaje de las matemáticas y el aprovechamiento del tiempo libre a partir del proyecto AfroMatematiquín, como una respuesta innovadora para reversar el bajo desempeño de los estudiantes.

En definitiva, la revisión de los diferentes estudios realizados refiere la importanciadelalúdicayel pensamiento aleatorio en los estudiantes, donde los aspectos pedagógicos y didácticos del docente son fundamentales para fortalecer desde las matemáticas dicho pensamiento, el cual muestra vacíos y evoca una problemática real en la práctica docente de una institución educativa ubicada en el municipio de Duitama, orientada por la pregunta: ¿De qué manera la aplicación de una estrategia lúdica contribuye al fortalecimiento de los procesos de pensamiento aleatorio y sistemas de datos en los estudiantes de los grados segundo y tercero? Se trata entonces de implementar una estrategia didáctica que permita fortalecer el concepto de este pensamiento en los estudiantes, no sin antes caracterizar las dificultades de los estudiantes de acuerdo con las teorías didácticas y pedagógicas actuales.

\section{Sustentos teóricos}

Teniendo en cuenta que el pensamiento aleatorio y sistemas de datos es un componente esencial en el mundo de las matemáticas, Araneda (2012) destaca que "El pensamiento aleatorio es la llave para adaptarse al mundo moderno, dinámico e impredecible” (p.4), su importancia en la actualidad respecto de la enseñanza de las matemáticas, se ha convertido en una gran reto ya que es una de las áreas fundamentales y de mayor envergadura en las instituciones educativas del país; donde se tiene en cuenta principalmente el pensamiento lógico y el numérico, dejando de lado las demás clases de pensamiento en especial el aleatorio y sistemas de datos.

Es necesario que el pensamiento aleatorio se desarrolle en forma significativa y dinámica, teniendo presente una propuesta didáctica atractiva para el estudiante; al respecto, el documento Estándares Básicos de Competencias (2006), menciona la necesidad de que "se establezcan conexiones con la vida del estudiante, la de sus familias, con las demás actividades de la institución educativa y, en particular, con las demás ciencias y con otros ámbitos de las matemáticas mismas" (p.70).
A la par, el trabajo de García Quintero (2012) planteo una estrategia didáctica basada en la lúdica para promover el aprendizaje de las matemáticas y el aprovechamiento del tiempo libre a partir del proyecto AfroMatematiquín, como una respuesta innovadora para reversar el bajo desempeño de los estudiantes. 
Explorar e interpretar los datos, relacionarlos con otros, conjeturar, buscar configuraciones cualitativas, tendencias, oscilaciones, tipos de crecimiento, buscar correlaciones, distinguir correlación de causalidad, calcular correlaciones y su significación, hacer inferencias cualitativas, diseños, pruebas de hipótesis, reinterpretar los datos, criticarlos, leer entre líneas, hacer simulaciones, saber que hay riesgos en las decisiones basadas en inferencias (p.31)
Retomando la afirmación de Araneda (2012), cuando se refiere a la trascendencia del componente aleatorio y su vital importancia en el aprendizaje de las matemáticas porque "al crear y resolver problemas de análisis combinatorio, los estudiantes estimulan el razonamiento lógico y la visión crítica de la realidad al no percibir la realidad como verdad absoluta, sino como un continuo investigar y descubrir" (p.4). Se evidencia la importancia del desarrollo del pensamiento aleatorio, como eje fundamental de la formación integral de los estudiantes, donde se replantee la manera como se está implementado este pensamiento al interior de las aulas de clase y el uso adecuado y pertinente de las metodologías, para que tanto docentes como estudiantes valoren lo fundamental de este pensamiento en su formación.

Con respecto a las características del pensamiento aleatorio, es preciso analizar que el pensamiento aleatorio y sistemas de datos, está presente en la ciencia, la cultura y sin lugar a duda en la forma de pensar cotidiana, este pensamiento ha forjado un camino en la matemática, al manejar acertadamente lo que está en la incertidumbre; además de señalar los aspectos más importantes que lo forman y que permiten relacionar las matemáticas con el carácter cambiante de la naturaleza y su relación con el avance de la ciencia y la tecnología.

Se resalta, entonces, que este tipo de pensamiento es tan importante porque favorece el desarrollo de habilidades mentales como son: observación, comparación, ordenación, clasificación, lógica, análisis; posibilitando de esta manera la interacción del estudiante con el mundo que lo rodea, comprendiéndolo y permitiendo la participación activa de él en la resolución acertada de diversas situaciones, se considera, como una actividad productiva y con sentido porque acerca a los estudiantes a la búsqueda de respuestas a preguntas sobre el mundo físico, usando la información necesaria para interpretarla, y posteriormente obtener las respuestas, que sirven de gran ayuda para la creación de nuevas hipótesis y búsqueda de actividades exploratorias muy enriquecedoras para los estudiantes.

Lo expuesto anteriormente también lo afirma Vasco citado en los Lineamientos curriculares para matemáticas (1998), cuando expone:

Explorar e interpretar los datos, relacionarlos con otros, conjeturar, buscar configuraciones cualitativas, tendencias, oscilaciones, tipos de crecimiento, buscar correlaciones, distinguir correlación de causalidad, calcular correlaciones y su significación, hacer inferencias cualitativas, diseños, pruebas de hipótesis, reinterpretar los datos, criticarlos, leer entre líneas, hacer simulaciones, saber que hay riesgos en las decisiones basadas en inferencias (p.31)

Por su parte en lo que se refiere al pensamiento aleatorio y al currículo, se muestran los estándares establecidos para los grados de primero a tercero en lo referente al pensamiento aleatorio de acuerdo al documento de Estándares Básicos de Competencias (MEN, 2006). 
Tabla 1. Estándares básicos de competencias en matemáticas

\begin{tabular}{l|l|l}
\hline \multicolumn{2}{c}{ PENSAMIENTO ALEATORIO Y SISTEMAS DE DATOS (Primero a Tercero) } \\
\hline & $\begin{array}{l}\text { Clasifico y organizo datos } \\
\text { de acuerdo con cualidades }\end{array}$ & $\begin{array}{l}\text { Describo situaciones o eventos a } \\
\text { partir de un conjunto de datos. }\end{array}$ \\
$\begin{array}{l}\text { Interpreto cualitativamente } \\
\text { datos referidosa situaciones } \\
\text { del entorno escolar. }\end{array}$ & $\begin{array}{l}\text { yablas. } \\
\text { tablas. los presento en }\end{array}$ & $\begin{array}{l}\text { Explico desde mi experiencia la } \\
\text { posibilidad o imposibilidad de } \\
\text { ocurrencia de eventos cotidianos. }\end{array}$ \\
$\begin{array}{l}\text { Identifico regularidades y } \\
\text { tendencias del conjunto de } \\
\text { datos. }\end{array}$ & $\begin{array}{l}\text { Represento datos relativos a } \\
\text { mi entorno usando objetos } \\
\text { concretos, pictogramas y } \\
\text { diagramas de barras. }\end{array}$ & $\begin{array}{l}\text { Predigo la posibilidad de } \\
\text { ocurrencia de un evento mayor } \\
\text { al otro. }\end{array}$ \\
& $\begin{array}{l}\text { Resuelvo y formulo } \\
\text { preguntas que requieran } \\
\text { para su solución coleccionar } \\
\text { y analizar datos del entorno } \\
\text { próximo. }\end{array}$ & \\
\hline
\end{tabular}

Nota: Estándares básicos de competencias en matemáticas (MEN, 2006, p.36)

Con base en lo anterior, es indispensable como docentes, fomentar situaciones de aplicación que involucren los conceptos aleatorios, dando lugar a situaciones de enseñanza orientadas al desarrollo de experiencias en función de este pensamiento, teniendo en cuenta que es factible que haya cambios y resultados inesperados que resultarían favorables, motivadores e interesantes para tomarlos como alternativas de ayuda y retroalimentación.

En busca del carácter didáctico para el desarrollo de la guía lúdica, Campos (2000) definelas estrategias de aprendizaje como los medios implementados por los docentes para mediar, facilitar, promover y organización mediante el uso de procesos que el estudiante desarrolla en pro de su aprendizaje, así como la estructura de las actividades que lo faciliten. Es decir, el apropiado desarrollo de estrategias de enseñanza y aprendizaje propende por la búsqueda de aprendizajes significativos y la construcción de conocimientos propicios que además de encaminar al estudiante hacia un adecuado proceso, también ofrece al docente diversas posibilidades de mejoramiento continuo en sus prácticas pedagógicas, fortaleciendo el uso de herramientas y espacios educativos creativos.

Por otra parte, Mosquera y Velasco (2008) describen que la estrategia didáctica puede ser determinada como "el conjunto de procedimientos, apoyados en técnicas de enseñanza, que tienen por objeto llevar a buen término la acción pedagógica del docente”(p.3), ya que gracias a este tipo de estrategias es posible fomentar procesos de autoaprendizaje, aprendizaje interactivo y aprendizaje colaborativo. 
la trascendencia del componente aleatorio, es de gran importancia para el aprendizaje de las matemáticas porque al crear y resolver problemas de análisis combinatorio, los estudiantes estimulan el razonamiento lógico y la visión crítica de la realidad al no percibir la realidad como verdad absoluta, sino como un continuo investigar y descubrir (p.4).
De igual manera, es importante considerar que la lúdica a través de la historia ha venido evolucionando potencialmente; y que día a día permite acercarse hacia la reflexión sobre su aplicación en la pedagogía, siendo el juego un nuevo facilitador de procesos de aprendizaje, pues como se observa, en la actualidad, es fundamental emplear la lúdica en los métodos de enseñanza, como una herramienta educativa que permite interactuar por medio de juegos o actividades lúdicas donde los estudiantes puedan acercarse al desarrollo de habilidades matemáticas.

Por esta razón es importante dinamizar las clases, tal vez hasta el punto de crear un espacio para y por la lúdica; sin dejar de lado la forma como los estudiantes deben aprender los conceptos e ideas del área de una forma significativa, generando un alto grado de motivación en ellos, lo cual les permita afianzar sus conocimientos y alcanzar los logros esperados en el ámbito escolar y el contexto donde se desenvuelven.

Ahora es el momento de explicar los aspectos teóricos y metodológicos relacionados con la lúdica como el principal artífice para el proceso de aprendizaje, porque su esencia está en el espacio dinámico y transformador. El elemento principal, del aprendizaje lúdico, es el juego, recurso educativo que debe ser aprovechado muy bien como uno de los instrumentos más importantes para el desarrollo integral del estudiante. Por ejemplo, para Zúñiga Benavides (1998) en su texto la pedagogía lúdica: una opción para comprender, centra una marcada diferencia entre la escuela de hoy y la que se debería tener, se pregunta qué tanto la escuela de hoy refuerza a los niños y niñas de forma integral, de tal manera que les permitirá alejarse del mundo cuadriculado que les ofrece la sociedad llena de normas que los aconductan, y que los moldean tanto como las comunidades lo desean.

Analizando lo expuesto por Guillermo Zúñiga es necesario hacer una reflexión consiente de la pedagogía actual, fortaleciendo la lúdica como la principal fuente de preguntas $y$ respuestas inmediatas a un mundo moderno y cambiante. Entonces, la lúdica claramente se puede tratar de una propuesta pedagógica, con diversidad de herramientas innovadoras que proporciona ámbitos adecuados de aprendizaje, trasciende los espacios del aula y propende por el desarrollo de pensamiento aleatorio y sistemas de datos, que es el pensamiento que se propone trabajar en esta propuesta investigativa, tal como lo afirma Araneda (2012),

la trascendencia del componente aleatorio, es de gran importancia para el aprendizaje de las matemáticas porque al crear y resolver problemas de análisis combinatorio, los estudiantes estimulan el razonamiento lógico y la visión crítica de la realidad al no percibir la realidad como verdad absoluta, sino como un continuo investigar y descubrir (p.4).

Es decir que la lúdica favorece el desarrollo de una variedad de propósitos, dentro del contexto de aprendizaje, pues poco a poco se 
construye autoconfianza e incrementa la motivación de los estudiantes. Es un método que muestra en forma eficaz lo significativo de aquello que se aprende, además el solo hecho de ser atractiva y motivadora, capta la atención de los estudiantes, es oportuno para el uso de los mecanismos y estrategias de aprendizaje.

\section{Desarrollo metodológico}

La investigación está enmarcada en una metodología de tipo cualitativo descriptivo la cual cuenta con objetivos predeterminados, un contexto objeto de estudio, características de los estudiantes, técnicas e instrumentos de recolección de información, procesos de interacción entre el investigador (docentes) y el objeto de estudio (educandos), evidencias recolectadas, análisis de la información, todas estas acciones encaminadas a mejorar la práctica a través de decisiones basadas en el análisis y la investigación y no sólo en intuiciones; como lo afirma Briones (2004), la investigación acción en el aula es realizada por el docente con el propósito de emplear conocimientos que obtengan en ese proceso la construcción de un determinado problema que se presenta en todos o en algunos de los estudiantes. De esta manera mejorar las prácticas pedagógicas de las instituciones en las cuales se aplica la investigación a partir de la comprensión de la realidad, a través de una cultura más reflexiva, sobre la relación existente entre los procesos, la teoría y la práctica producto de la labor educativa.
Con respecto al desarrollo de la propuesta metodológica se realiza siguiendo un modelo en espiral en ciclos sucesivos que incluyen; diagnóstico, desarrollo y validación por medio de la planificación, acción, observación, reflexión y valoración, cuenta con objetivos predeterminados, un contexto objeto de estudio basado procesos de interacción que permiten mantener un clima armónico y de mutua confianza en el aula durante todo el proceso investigativo, de esta manera la estructura metodológica de la investigación acción (IA) para Kemmis, citado por Latorre (2007) " es una forma de indagación auto reflexiva realizada por quienes participan.” (p. 24) que se basa en ciclos que luego de su estandarización se pueden convertir en espirales de acción.

La institución educativa donde se realiza la investigación está ubicada en el casco urbano de la ciudad de Duitama, en el departamento de Boyacá y cuenta con una población de carácter mixto de 1800 estudiantes; la institución al tener un carácter técnico, orienta áreas específicas de la modalidad, en las cuales priman los contenidos e intensidades horarias requeridas por las especialidades, así como los parámetros y contenidos curriculares con un énfasis de modalidad técnica, es decir que los estudiantes se forman como líderes técnicos en diferentes campos, lo que les permite ser partícipes del desarrollo socioeconómico de la región.

Entre tanto, los estudiantes de grado segundo y tercero de educación básica 
primaria que conforman la unidad de análisis tienen edades que oscilan entre los siete y los nueve años; es importante resaltar que en el grado segundo hay un niño autista y un estudiante nuevo; donde se observan familias que brindan poca atención y apoyo a los procesos académicos de sus hijos, por factores asociados a las actividades laborales, delegando esta responsabilidad a otras personas. Es así como las dificultades de aprendizaje en algunos de estos estudiantes, se presentan probablemente se debido a la escasa estimulación en el desarrollo de habilidades cognoscitivas, falta de acompañamiento familiar, poca formación en hábitos de estudio, sumado al desinterés hacia los procesos educativos.

Además se observa que la visión del Proyecto Educativo Institucional (PEI) está encaminada hacia la búsqueda del liderazgo en la formación de estudiantes técnicos industriales, humanistas y científicos, que transformen el entorno social $y$ productivo del medio local, regional y nacional, esta visión es bastante clara y precisa, sin embargo, algunos estudiantes se les dificulta proyectar este liderazgo en la creación de empresa, y enfocan su proyecto de vida hacia otros campos laborales diferentes al que se aspira en la parte técnica y su proyecto de vida es encaminado hacia otros espacios y otras ramas.

Complementando lo anterior, se observa en algunos estudiantes presentan diversas dificultades de aprendizaje, reflejadas en el bajo rendimiento académico, en el ambiente educativo, en la posible desventaja frente a los pares, en las actitudes poco motivantes; esto unido a la falta de compromiso y acompañamiento familiar por parte de algunos padres de familia, que ven a la institución más como un lugar de cuidado para sus hijos y no como formadora de principios, valores y ambientes de aprendizaje; al igual, que se evidencia que algunos grupos familiares no favorecen el desarrollo personal y social del estudiante, familias que se desenvuelven en un ambiente donde faltan pautas de crianza y hábitos de estudio, lo cual, poco favorece el desempeño escolar.

De igual forma se analizaron los resultados del Índice Sintético de Calidad (ISCE) y las pruebas externas e internas, donde se evidencian alcances satisfactorios, los cuales conducen hacia la reflexión y el compromiso para seguir trabajando hacia la excelencia, ideal para continuar con una mejor calidad en el nivel educativo de la institución. Es así como es apremiante potenciar las acciones de mejoramiento, en los componentes de desempeño y progreso, tales como el seguimiento continuo de aprendizajes significativos buscando mejorar el área de matemáticas, mediante estrategias enseñanza que fortalezcan principalmente el pensamiento aleatorio y sistemas de datos, con un enfoque de evaluación formativa; siendo esto un factor que prevalece en la mejora de los resultados en pruebas internas y externas. 
Para la recolección de información en las diferentes fases de la investigación y en concordancia con el enfoque y tipo de investigación, se está trabajando con el diario de campo, en él se registrarán las narraciones de aquello que es percibido y escuchado por los docentes investigadores en el aula de clase. Como lo señala Bonilla y Rodríguez (1997),

el diario de campo debe permitirle al investigador un monitoreo permanente del proceso de observación. Puede ser especialmente útil [...] al investigador en él se toma nota de aspectos que considere importantes para organizar, analizar e interpretar la información que está recogiendo (p.129).

Entonces, el diario de campo es de vital importancia ya que establece la relación entre lo teórico y lo práctico, asimismo, la observación participante es una técnica de investigación que necesita de una planeación para abordar un objeto de estudio a través de un trabajo de campo (práctica), esto con el fin de reflexionar profundamente para proyectarlo al quehacer docente con criterios veraces que soporten esa práctica.

De otro lado, se aplicó un taller diagnóstico, considerado como un espacio de trabajo compartido por dos o hasta tres grupos focales simultáneamente. Técnica de particular importancia porque brinda la posibilidad de abordar, desde una perspectiva integral y participativa, el problema que requiere algún cambio. Es decir, a partir del diagnóstico de la situación identificando, valorando y formulando planes de acción.
En relación con lo anterior, es necesario puntualizar que una de las técnicas más representativas en la presente investigación es la observación participante, siendo esta una acción explicita que requiere de atención, detalle y concentración para seleccionar aspectos concretos del desarrollo de estrategias didácticas para concebir, analizar y valorar el proceso del fortalecimiento del pensamiento aleatorio y sistemas de datos en el área de matemáticas en los estudiantes de segundo y tercero de la institución.

\section{Resultados fase diagnóstica}

El taller diagnóstico para el grado segundo presenta cuatro aspectos a saber: en el primer se dio a conocer una situación y los niños debían seleccionar la información suministrada de acuerdo con las categorías de cantidad y calidad; seguidamente organizaban los datos suministrados en una tabla de frecuencias y en un diagrama de barras. Un segundo aspecto que se tiene en cuenta es el número de combinaciones que pueden hacer los niños al consumir cuatro alimentos sólidos con dos líquidos. El tercero determina los posibles arreglos que hicieron los niños con el uso de una vocal y una consonante; la prueba culminó con algunas probabilidades a partir del juego con una ruleta de colores.

Los resultados de la prueba diagnóstica en el grado segundo fueron los siguientes: en el primer aspecto 24 niños establecen acertadamente las categorías de cantidad y de calidad suministradas por el texto, es decir los el diario de campo debe permitirle al investigador un monitoreo permanente del proceso de observación. Puede ser especialmente útil [...] al investigador en él se toma nota de aspectos que considere importantes para organizar, analizar e interpretar la información que está recogiendo (p.129). 
niños tienen claridad en diferenciar la cantidad de un determinado articulo con respecto a la calidad del mismo. En el mismo sentido cinco niños interpretan de forma incompleta la información suministrada, en tanto que cuatro niños presentan confusión en sus representaciones. Caso contrario sucede cuando les solicitan que con la información dada, completen la tabla de frecuencia y la representen en barras verticales, solo cuatro niños identifican que las características las debían ubicar en sentido horizontal, mientras que la cantidad la debían ubicar en sentido vertical; ocho niños logran ubicar las características en la barra horizontal, dejando la vertical vacía; 11 niños no logran identificar la información ni en sentido horizontal ni vertical; cuatro niños no poseen sentido de ubicación $\mathrm{y}$, mientras que seis de ellos no hacen nada.

En el segundo aspecto el niño debía establecer las posibles combinaciones que se podían hacer con cuatro alimentos sólidos y dos líquidos, la solución pedía colocar la cantidad de combinaciones y escribirlas. Cinco niños realizan el ejercicio de manera acertada, 11 hacen las combinaciones, pero no escriben la cantidad en el cuadro correspondiente; de esta información se infiere que los niños están en capacidad de hacer combinaciones entre dos magnitudes sin importar sus características. Once niños hacen las posibles combinaciones, pero en el recuadro donde debían completar con la cantidad, escriben inconsistencias que no corresponden a lo solicitado y, seis no contestaron absolutamente nada.
El tercer aspecto consistía en escribir posibles arreglos que los niños podían hacer con una vocal y una consonante, el instrumento proporciona dos consonantes y tres vocales. 27 niños escriben arreglos de dos vocales y dos consonantes, de dos vocales y una consonante o de dos consonantes y una vocal, de lo cual se puede deducir que los estudiantes no comprenden los enunciados suministrados en el instrumento; seis de los 33 niños no escribieron nada.

Finalmente, en el cuarto punto se presenta el dibujo de una ruleta con ocho secciones identificadas con diferentes colores, los niños debían establecer la probabilidad de ocurrencia de un suceso, que no sucediera el suceso, y cual suceso era poco probable que sucediera. Además, con la información suministrada establecer la probabilidad de cada color partiendo de la definición "parte de un todo". A la probabilidad de que no ocurra un suceso solo contestaron 14 niños; a la posibilidad de que es poco probable que gane solo contestaron acertadamente siete estudiantes y a la pregunta ¿quién tiene más probabilidad de ganar?, contestan acertadamente 23 estudiantes. En el segundo aspecto se obtiene la siguiente información 25 estudiantes asimilan la relación existente entre la probabilidad de un suceso y su representación numérica como parte de un todo. 8 estudiantes no poseen la mencionada competencia.

Por su parte el taller diagnóstico para el grado tercero presenta cuatro aspectos, en el primer se dio a 
conocer una situación y los niños debían seleccionar la información suministrada de acuerdo con variables cuantitativas y variables cualitativas; los demás aspectos son similares a los presentados en el grado segundo.

De los 35 estudiantes del grado tercero de básica primaria 28 contestan acertadamente, es decir presentan claridad en diferenciar la variable cuantitativa de la variable cualitativa cuando les suministran una información; tres de los niños confunden dichas variables, para otros tres son iguales y dos de ellos no identifican lo cualitativo ni lo cuantitativo.

En la representación de los datos en tablas de frecuencia y en diagrama de barras sucede algo muy similar al punto anterior, 29 de los niños completan la tabla y la representan acertadamente en el diagrama de barras sin tener en cuenta las características de los ejes horizontal y vertical. Los seis niños restantes no contestan la pregunta, la dejan en blanco.

En el caso de las posibles combinaciones existentes entre las cuatro comidas y las dos bebidas, 22 niños contestan acertadamente y escriben las posibles combinaciones, los 13 restantes contestan incoherencias, ejemplo de ello que existen cuatro, siete, 12 o 16 combinaciones, situación que conduce a inferir que los niños no poseen conocimiento del significado de combinación cuando se trata de dos o más objetos.
En la tercera situación donde los niños debían escribir los posibles arreglos con dos letras, una consonante y una vocal, solo seis niños no entendieron la actividad y por tal razón contestaron de manera errónea. En la cuarta situación a la probabilidad de que no ocurra un suceso contestaron acertadamente 25 niños; a la posibilidad de que es poco probable que gane contestaron acertadamente 27 estudiantes y a la pregunta ¿quién tiene más probabilidad de ganar?, contestan acertadamente 30 estudiantes. En el segundo aspecto 32 estudiantes asimilan la relación existente entre la probabilidad de un suceso y su representación numérica como parte de un todo.

\section{Conclusiones}

Una vez aplicado el taller diagnóstico y analizada la información se puede establecer que, aunque la prueba para los niños de segundo y tercero guarda similitud, resulta más complejo el desarrollo para los de segundo, sin embargo, en el primer aspecto, los resultados evidencian que los niños de segundo establecen con facilidad los conceptos de cantidad y calidad, mientras que a los de tercero la palabra "variable" en el enunciado les complica diferenciar lo cualitativo de lo cuantitativo. Los dos grupos presentan dificultades similares al momento de relacionar la tabla de frecuencias con el diseño de la gráfica de barras, no colocan las cantidades en la barra vertical, ni las características en la horizontal; y en los dos grupos se evidencian estudiantes con problemas de ubicación espacial porque no saben 
cómo ubicar la información recogida o suministrada en la tabla de frecuencias.

De igual manera los dos grupos presentan problemas cuando se trata de realizar combinaciones con dos o más cantidades, para el caso el número de combinaciones se obtiene de multiplicar el número de comidas por el número de bebidas, es decir cuatro comidas por dos bebidas, o sea ocho posibilidades. Los estudiantes que no contestan acertadamente a la pregunta y más aún que no describen las posibilidades que existen, en los niños de segundo se infiere que no relacionan el número de posibilidades con el resultado de multiplicar los dos tipos de alimentos y en los de tercero puede ser porque no entienden el enunciado de la pregunta o porque no saben multiplicar.

Algo similar sucede con el punto tres, como son tres vocales y dos consonantes, los niños debían realizar la multiplicación y dar a conocer las posibilidades mediante la escritura de las mismas. Para los niños de grados segundo puede presentar cierto grado de dificultad por la interpretación del enunciado, pero para los niños del grado tercero se concluye que no comprenden lo que les están preguntando o simplemente no saben relacionar las dos cantidades que se les presenta.

Para finalizar esta discusión, en el cuarto punto, a partir del dibujo de una ruleta y de la información que allí se suministra el niño debe contestar a las preguntas de acuerdo con la probabilidad de la existencia de un determinado suceso. Se reitera que a los niños de segundo grado de básica se les dificulta por el grado de comprensión lector que ellos poseen y porque para ese nivel el concepto de fracción como parte de un todo aún no se les ha suministrado. De igual forma se debe aclarar que expresiones como "poco probable", "mayor probabilidad" y "complete la probabilidad" en niños de segundo tienen un grado de dificultad mayor que en niños de tercero, sin embargo, al analizar las respuestas de uno y otro grado, se percibe una similitud en la manera de analizar la situación.

\section{Referencias}

Antonio, L. (2007). La investigación-acción. Conocer y cambiar la práctica educativa. Barcelona, Editorial Graó, de IRIF, SL,

Araneda, A. (2012) Reflexión: Pensamiento aleatorio. Las matemáticas convierten lo invisible en visible.

Bonilla, E., \& Rodríguez, P. (1997). Más allá del dilema de los métodos. La investigación en ciencias sociales. Colombia, Editorial Norma.

Briones, G. (2004). La investigación en el aula y en la escuela, módulo 2. Convenio Andrés Bello. Editorial Gente Nueva. Bogotá DC. 
Camacho, M. (2015). La implementación de actividades ludico-pedagogicos para fortalecer el rendimiento académico en el área de matemáticas. Tunja.

Campos, Y. (2000). Estrategias didácticas apoyadas en tecnología. México: Secretaría de Educación Pública. Accesible en http://wrwe. camposc. net/Orepositorio/libros/ estrategias/libroEstrategias. html.

Ministerio de Educación Nacional. (2006). Estándares básicos de competencias en lenguaje, matemáticas, ciencias y ciudadanas. Santa fé de Bogotá: Ministerio de Educación Nacional.

Ministerio de Educación Nacional. (2006). Todos a aprender. Introducción al pensamiento aleatorio y sistemas de datos. Bogotá, Colombia.

Ministerio De Educación Nacional (2008). Lineamientos curriculares para matemáticas. Santafé de Bogotá.

Renteria Maturana, L., López Botero, F. M., \& Vergara Mazo, F. A. (2016). El aprendizaje de las operaciones básicas matemáticas en educación primaria, mediado por ambientes virtuales de aprendizaje: el caso de la IE Pascual Correa Flórez del Municipio de Amagá, IE San Luis del Municipio de San Luis y Centro Educativo Rural El Edén del Municipio de Granada.

Uribe, C. E. V., de Losada, M. F., Castañeda, J. C., \& Márquez, R. L. (2016). Consideraciones sobre la enseñanza de la matemática en el ciclo diversificado colombiano. Cuadernos de Investigación y Formación en Educación Matemática, (7).

Velasco, M., \& Mosquera, F. (2008). Estrategias didácticas para el aprendizaje colaborativo. Consultado, 3, 3.

Zúñiga, M., \& Gómez, R. Construyendo una pedagogía para la paz desde el proyecto sobre violencia y convivencia en Cali: nuevos escenarios de la educación popular. Construyendo una Pedagogía para la Paz desde el Proyecto sobre Violencia y Conviencia en Cali: Nuevos Escenarios de la Educación Popular. 